\title{
SDR Transceiver Architecture on FPGA: A Review
}

\author{
Shreevani. $\mathrm{C}^{1}$, Ashoka. $\mathrm{A}^{2}$, Praveen. $\mathrm{J}^{3}$, Raghavendra Rao. $\mathrm{A}^{4}$ \\ M.Tech, $2^{\text {nd }}$ year, VLSI Design and Embedded Systems, ECE Dept., A.I.E.T, Mijar, Moodbidri, Karnataka, India ${ }^{1}$ \\ Assistant Professor, ECE Department, A.I.E.T, Mijar, Moodbidri, Karnataka, India ${ }^{2}$ \\ Senior Associate Professor, ECE Department, A.I.E.T, Mijar, Moodbidri, Karnataka, India ${ }^{3,4}$
}

\begin{abstract}
Since from 1980's, the US military has been working on SDR to increase the ease and flexibility of communication within and between the services. Different forces present at that time used dozens of different radios. Most of the radios could only communicate with the other radios of same kind. Software Defined Radio (SDR) offered the promise of not only interoperating with all existing radios, but also allowing those radios to communicate with each other. Just as important, SDR has enabled future communication systems to be implemented on already deployed hardware. Software Defined Radio (SDR) is a radio communication system where components that have been typically implemented on hardware are instead implemented by means of software on a personal computer or embedded system. While the Software defined radio is not new concept, the rapidly evolving capabilities of digital electronics render practical many processes which used to be only theoretically possible. A software defined radio (SDR) allows for digital communication system to easily adopt more sophisticated coding and modulation techniques, which is extremely important in meeting the ever increasing demands of the wireless communication [7].
\end{abstract}

Keywords: QPSK, Power efficiency, Spectral efficiency, Synchronisation.

\section{INTRODUCTION}

Traditional radio communication systems needed a lot of hardware components such as detector, demodulator, filter, etc. Which made the platform cost very much high for the undergraduate level study and research. But the laboratory exercises and studies on that aspect are necessary for related teachers and students. It is possible to implement the radio communication process with software using software defined radio. Comparing with traditional radio communication system, SDR omits all the hardware and replaces them by pure software. This solution can also gives a great advantage in flexibility, because SDR receiver is able to decode all the signals. A software defined radio would be able to transmit and receive signals of any frequency, power level, bandwidth, and modulation techniques.

A software defined radio (SDR) allows the digital communication systems to easily adopt most sophisticated coding and modulation technologies, which is extremely important to meet the ever increasing demands of the wireless communication industry.

The signals with high frequency can be transmitted in radio communication system. On one hand, only the signal with high frequency can be transmitted over a long distance; on the other hand, the height of the antenna has a strong relationship with the signal frequency. The lower the frequency the higher the antenna is. Thus to transmit low frequency signal may require a very high antenna which ever cannot be made out. Whenever the signal with low frequency needs to be transmitted it is necessary and important to modulate it to a high frequency signal. Only in this way can the signal be transmitted in the radio communication system.

Modulation techniques are used in SDR. Modulation is a process in which some characteristics of the carrier wave are varied with respect to modulated signal. Three major kinds of modulation technologies are used in radio communication system. They are Amplitude-Shift Keying (ASK), Frequency-Shift Keying (FSK) and Phase-Shift Keying (PSK). In recent years BPSK and QPSK are being used. BPSK stands for Binary Phase Keying which is the simplest PSK. In BPSK the carrier signals phase varies between two values according to the modulating signal. To improve efficiency QPSK modulation is being used. Quadrature Phase Shift keying is another phase shift modulation method which is little more complex than BPSK. It has twice bandwidth efficiency of BPSK [1].

\section{HISTORY OF SOFTWARE DEFINED RADIO}

The term "digital receiver" was coined in 1970 by a researcher. The term "software radio" was coined in 1984 by a team at the garland, Texas Division of baseband receiver. In 1984 software radio was a digital baseband receiver that provided programmable interference cancellation and demodulation for broadband signals, typically with thousands of adaptive filter taps, using multiple array processors accessing shared memory.

In 1991, joe Mitola independently reinvented the term software radio for a plan to build a GSM base station. SPEAKeasy, the military software radio was formulated by Wayne Bonser. Mitola was introduced as "godfather" of software radio in 1997 at first international conference on Software radios. Perhaps the first software based radio transceiver was designed and implemented by Peter Hoeher and Helmuth Lang at the German Aerospace Research Establishment. 
The term "software defined radio" was coined in 1995 by by varying one or more features of a carrier signal. When Stephen Blust in 1996; software-defined radios have their the receiver receives a modulated signal, it has to process origins in the defense sector since from 1970s in both the the modulated carrier signal and get the original U.S and Europe. The primary goal of the SpeakEasy information; this process is demodulation. Its function is project was to use programmable processing to emulate opposite to that of modulation.

more than 10 existing military radios, operating in frequency bands between 2 and $2000 \mathrm{MHz}$. Another SPEAKeasy design goal was to be able to easily incorporate new coding and modulation standards in the future, so that military communications can keep pace with advances in coding and modulation techniques.

\section{SPEAKeasy phase I}

From 1990 to 1995 , the goal of the SPEAKeasy program was to demonstrate a radio for the U.S Air force tactical ground air control party that could operate from $2 \mathrm{MHz}$ to $2 \mathrm{GHz}$, and thus could interoperate with ground force radios, Air force radios, Naval Radios and satellites.

The basic arrangements of the radio receiver used an antenna feeding an amplifier and down-converter feeding an automatic gain control, which fed an analog to digital converter that was on a computer bus with a lot of digital signal processors. The transmitter had digital to analog converters on the PCI bus feeding an up converter that led to a power amplifier and antenna. The very wide frequency range was divided into a few sub-bands with different analog radio technologies feeding the same analog to digital converters. This has since become a standard design scheme for wide band software radios.

SPEAKeasy phase II

The goal of this program was to get a more quickly reconfigurable architecture. The secondary goals were to make it smaller, cheaper and weigh less. The software architecture identified standard interfaces for different modules of the radio: "radio frequency control" to manage the analog parts of the radio, "modern control" resources for modulation and demodulation schemes [4].

\section{BASIC CONCEPTS OF MODULATION AND DEMODULATION}

In radio communication system the signal with high frequency has been transmitted. On other hand, only the signal with high frequency can be transmitted over a long distance; on the other hand the height of antenna has a strong relationship with signal frequency. The lower the frequency is the higher the antenna is. Thus to transmit low frequency signal may require a very high antenna which even cannot be made out. Whenever the signal with low frequency needs to be transmitted it is necessary and important to modulate it to a high frequency signal. Only in this way can the signal be transmitted in the radio communication system.

The signal used to carry message is called carrier signal; typically it is a high frequency sinusoid or cosine waveform. The carrier signal can be transmitted via the air over a long distance. The process of making the radio frequency carrier signal carry the information signal with low frequency is modulation. Modulation can be realized

\section{A. Analog Modulation and Demodulation}

The modulation is the process of varying one or more features of a carrier signal. If the modulating signal is analog and the variations for the parameters of carrier signal based on the modulating signal is continuous, the modulation is treated as analog modulation. The parameters can be changed in carrier signal are amplitude and angle, while the angle contains frequency $\omega$ and phase $\theta$. When the amplitude of carrier signal varies as the modulating signal, it is amplitude modulation (AM); if the other two parameters are changed, it is called frequency modulation (FM) and phase modulation (PM) respectively.

\section{B. Digital Modulation and Demodulation}

The modulation process is digital modulation when the modulating signal is digital. Compared with analog modulation, digital modulation has a lot of advantages, for example greater noise immunity, greater security, etc.. Similar with analog modulation, there are three features can be modulated on carrier by digital information. Thus three major kinds of digital modulation technologies are used in radio communication system. They are AmplitudeShift Keying (ASK), Frequency-Shift Keying (FSK) and Phase-Shift Keying (PSK).

BPSK stands for Binary Phase-Shift Keying which is the simplest PSK. In BPSK the carrier signals phase varies between two values according to the modulating signal. BPSK is also called 2-PSK for the two values has $180^{\circ}$ difference. The method to modulate carrier is when the signal is bit 1 , the carrier's phase is one value; while when the bit is 0 , carrier's phase changes to another value. The pair of phase 0 and $\pi$ is used in this project. Suppose carrier signal is

$$
\mathrm{y} 1=\cos \left(2 \pi \mathrm{f}_{\mathrm{c}} \mathrm{t}\right)
$$

Phase of this carrier is 0 , from mathematic knowledge, it can be known that

$$
\mathrm{y} 2=\cos \left(2 \pi \mathrm{f}_{\mathrm{c}} \mathrm{t}+\pi\right)=-\cos \left(2 \pi \mathrm{f}_{\mathrm{c}} \mathrm{t}\right)=-\mathrm{y} 1
$$

Equation (2) offers the method to modulate the signal. When signal is bit 1 , BPSK signal is $y 1$, otherwise it's $\mathrm{y} 1$.

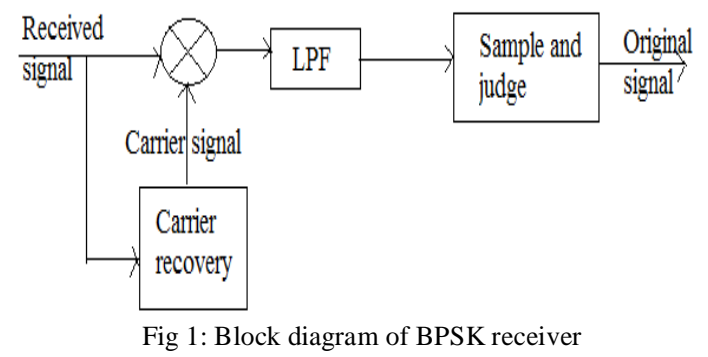

In theoretical, received BPSK signal should be

$\mathrm{r}(\mathrm{t})=\mathrm{A} \cos \left(2 \pi \mathrm{f}_{\mathrm{c}} \mathrm{t}+\mathrm{k} \pi\right) ;(\mathrm{k}=0,1)$ 
In this situation the carrier signal used to demodulate Asraf Mohammed Moubark et al [3], proposed that received signal can be as the same as the transmitter's modulation is a key feature commonly used in wireless process of carrier recovery can be omitted. But in reality, communication for data transmission and to minimize received signals phase is not only 0 or pi; it may have a phase shift $\varphi$. In this situation, received BPSK signal is

$$
\mathrm{r}(\mathrm{t})=\mathrm{A} \cos \left(2 \pi \mathrm{f}_{\mathrm{c}} \mathrm{t}+\mathrm{k} \pi+\varphi\right) ;(\mathrm{k}=0,1)
$$

The demodulation result will be wrong if receiver still use the same carrier recovery signal. To handle this problem carrier recovery circuit is used, which means to get the carrier signal from the received signal itself. The process of carrier recovery is shown in fig. 2 which is carrier recovery process.

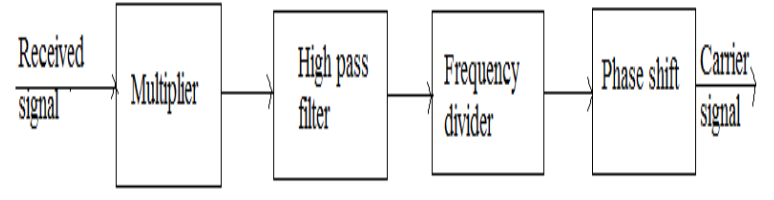

Fig : 2 Process of carrier recovery

Dividing this signals frequency to be half through a low pass filter, the carrier signal can be recovered to

$$
\begin{aligned}
& \mathrm{y}=\frac{1}{2} A^{2} \cos \left(2 \pi \mathrm{f}_{\mathrm{c}} \mathrm{t}+2 \varphi\right) \\
& \text { Carrier }=\frac{1}{2} A^{2} \cos \left(2 \pi \mathrm{f}_{\mathrm{c}} \mathrm{t}+\varphi\right)
\end{aligned}
$$

Receiver can multiply the received signal with this carrier signal to demodulate it.

Quadrature Phase-shift keying is another phase-shift modulation method which is a little more complex than BPSK. It has twice the bandwidth efficiency of BPSK, since two bits are transmitted in a single modulation symbol [5].

\section{REVIEW WORK}

Chris dick et al [2], proposed the carrier synchronization in SDRs using FPGA based signal processors. The carrier synchronization in SDRs provided overview of carrier recovery techniques for QPSK and QAM modulation schemes on the design and FPGA. It examines maximum likelihood carrier phase synchronization for QAM (Quadrature amplitude modulation) based SDR personalities. The FPGA device utilization and performance for a carrier recovery circuit using a look-up table approach are presented. The most complex component in the loop is the phase detector. Since the phase of QPSK or QAM signals is data dependent, the phase detector must strip the modulation from the received signal and produce a signal proportional to the phase difference between the local generated quadrature carriers and those of the received signal. The implementation of the high-performance digital communication systems has been made possible by advances in semiconductor process technology in the form of application specific standard parts, full custom silicon chips, instruction set based digital signal processors (DSPs) and high performance general-purpose processors (GPP). The phase error is computed by comparing the phase difference between the received signal and the closest constellation point. antenna design. QPSK (Quadrature Phase Shift Keying) is one type of digital modulation technique used to transfer the baseband data wirelessly in much efficient way compare to other modulation techniques. Conventional QPSK modulator operates by separation of baseband data into $i$ and $q$ phases and then add them to produce QPSK signal. The process of generating sine and cosine carrier wave to produce $\mathrm{i}$ and $\mathrm{q}$ phases consume high power. For better efficiency in power consumption and area utilization, 2 new types of QPSK modulator proposed. The proposed method will eliminate the generation of 2 phases and will produce the QPSK output based on stored data in RAM.

Shriram K Vasudevan et al [4], developed a model of a Software defined Radio using SIMULINK tool to implement the IEEE 802.11 standard and the Bluetooth standard. The main aim was to build various protocols for WLAN and the Bluetooth standards and to demonstrate their functionality. This includes implementation of IEEE 802.11a standard for the WLAN and basic core protocols for the Bluetooth. To develop a model of a Software Defined Radio which supports the IEEE 802.11a standard and Bluetooth standard using the SIMULINK tool, to implement all the main protocol stacks for WLAN and Bluetooth Protocols and verify their functionality, to transmit the data as frames using the format specified for the standard, to compress the binary data using source coding algorithm and also encode the data for Forward Error Correction (FEC). to modulate the binary stream of data using the BPSK modulation for WLAN mode which supports the basic transfer mode with $6 \mathrm{Mbps}$ speed, for the Bluetooth standard the basic modulation scheme used is GPSK and Frequency hopping spread spectrum is used, to implement algorithm for WLAN security and also CRC-16 for protecting the integrity of the data, to implement point to point communication and data transfer for the Bluetooth standard, to implement CSMA/CA technique and also implement different basic functions such as packet routing, Authentication, Request to transmit etc. for the WLAN standard.

Ziyi feng et al [5], from university of applied sciences implemented software defined radio using MATLAB to design a radio communication system in audio band using audio devices such as a sound card. The idea of SDR is to use ultra-high speed sampling and ADC/DAC modules directly measure the received radio signal and decode whatever it contains. The aim was to study and understand SDR using MATLAB in audio frequency band. Both analog and digital modulation and demodulation methods are studied. This will employ one desktop PC with MATLAB installed and simulates the radio communication process. Two Matlab sessions are executed at the same time, one as transmitter, and the other as receiver. When the project works, the signal is produced in transmitter and transmitted as sound after 
modulated. Then the receiver will receive the signal by recording it. The information can be obtained after demodulation.

Anton S. Rodriguez et al [6], proposed that softwaredefined radios (SDR) provide a versatile wireless communication solution for a wide range of applications, including cellular telephones, global positioning systems, and military grade communications. The SDR is applicable in nearly any wireless communication system and when implemented on a Field Programmable Gate Array. The SDR is a very cost-effective system in many ways. Since all hardware is physically programmed using software, re-design becomes relatively simple. Rather than discarding old hardware, the SDR is simply reprogrammed, updated and loaded back onto the FPGA, saving both time and money. The SDR also provides a capability for high quality communication without a need for expensive broadcasting equipment. In addition to its cost benefits, the SDR is also a very powerful and flexible system. In wireless communication, this means faster data rates and highly configurable modulation technology. Quadrature phase shift keying (QPSK) is a modulation scheme which sends a pair of bits per symbol, increasing data rate by a factor of two. A typical problem in QPSK and in wireless communication is carrier synchronization, or the synchronization of the oscillator at the receiver with the oscillator at the transmitter. In order to do so, a phaselocked loop circuit must be appended to the receiver. This provides the local oscillator at the receiver with a frequency adjustment. However, once this correction is made, a static phase error called phase ambiguity will still exist.

\section{IV.QPSK THEORY}

Quadrature phase shift keying (QPSK) is a digital modulation scheme where each symbol is represented by a pair of bits, increasing data rate by a factor of two in a given bandwidth. Higher modulation schemes, such as 8PSK and 16-PSK, sends three or four of bits per symbol to increase data rates even further. A typical concern in wireless communication using QPSK modulation is carrier synchronization at the receiver and hence requires a phase lock loop based carrier recovery technique at the receiver.

For transmission to and from a satellite, the information (baseband digital signal) must be modulated onto a microwave carrier. In general, the digital baseband signals may be multi-level (M-ary), requiring multilevel modulation methods. With QPSK, the binary data is converted into 2-bit symbols I, Q which are then used to phase modulate the carrier. Since four combinations containing 2 bits are possible from binary information (logical $1 \mathrm{~s}$ and $0 \mathrm{~s}$ ), the carrier phase can be shifted to one of the four states and transmitted. The mathematical representation of the transmitted signal, $s(t)$ can be written as

$s(t)=I(t) \cos \left(2 \pi f_{0} t\right)-Q(t) \sin \left(2 \pi f_{0} t\right)=\cos \left(2 \pi f_{0} t+\phi(t)\right)$

Here I,Q $€\{1 / \sqrt{ } 2,-1 / \sqrt{ } 2\}$ and $f_{0}$ denotes the carrier frequency and the vectors I, Q carry one bit information each $2 \pi f_{c} t$.

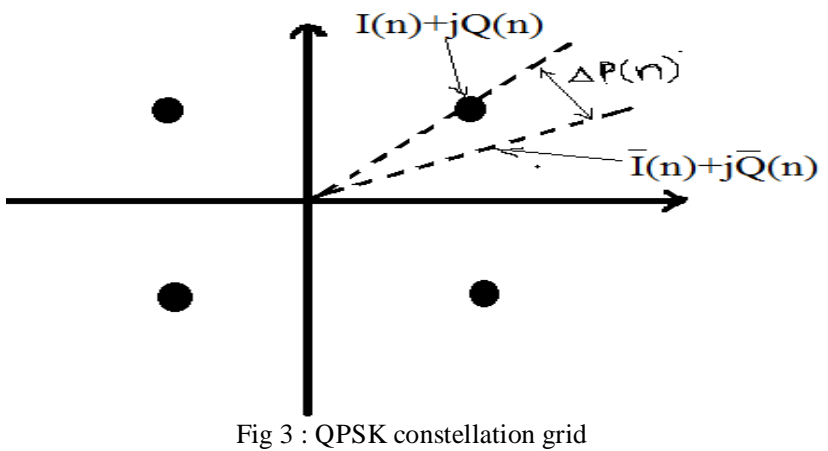

The received signal is demodulated and the decoded data can be represented as constellation diagram on an $\mathrm{x}-\mathrm{y}$ plane in terms of symbols at 45, 135, 225 and 315 degrees respectively.

Each symbol in the constellation represents two bits of information that are decoded based on their position in the constellation. Performance of any radio system depends on the efficiency of the modulation scheme used. The two most important factors that decide the overall efficiency of the modulation scheme are power efficiency and bandwidth efficiency. Power efficiency is the ability of the modulation technique to preserve the quality (e.g. BER) of the signal with minimal signal power. It is defined as the ratio of signal energy per bit to noise spectral density $(\mathrm{Eb} / \mathrm{No})$ required to achieve a particular BER. Bandwidth efficiency is the ability of the modulation technique to transfer more data at the given bandwidth, which decides the symbol/channel capacity. It is defined as the ratio of data rate in bits per second to allocated bandwidth in Hertz $(\mathrm{R} / \mathrm{B})$. There exists a fundamental trade off in any communication systems between the power efficiency and bandwidth efficiency as one can be achieved only at the expense of the other.

We can represent QPSK signals by:

$$
\mathrm{s}(\mathrm{t})=\frac{1}{\sqrt{2}} \mathrm{~d}_{\mathrm{I}}(\mathrm{t}) \cos \left(2 \pi \mathrm{f}_{\mathrm{c}} \mathrm{t}\right)+\frac{1}{\sqrt{2}} \mathrm{~d}_{\mathrm{Q}}(\mathrm{t}) \sin \left(2 \pi \mathrm{f}_{\mathrm{c}} \mathrm{t}\right)
$$

Two BPSK modulated signals combined together to represent a QPSK signal by using two orthogonal carrier signals. One is given by $\cos \left(2 \pi f_{c} t\right)$ and the other is given by $\operatorname{Sin}\left(2 \pi f_{c} t\right)$.

\section{CONCLUSION}

The SDR is an emerging class of very cost effective, low power system since hardware is physically programmed using software, re-design and reuse becomes relatively simple. Any change in the requirement specifications, the SDR is reprogrammed, updated and loaded back onto the FPGA, saving both cost and time instead of discarding old hardware. The SDR is also emerging as a popular platform. Software defined radios constitutes a versatile platform for wireless communication solutions like cellular, global positioning systems and military grade communications. The terminology of hybrid solution is also applicable to SDR approach as it applies to any wireless communication system implemented on Field programmable Gate Array (FPGA) in conjunction with requisite RF system. 


\section{REFERENCES}

[1] Shahana K, Ravi Kumar Gupta, K S Parikh,"SDR implementation of low frequency trans-receiver on FPGA".

[2] Chris Dick, Fred Harris, and Michael Rice, "The FPGA implementation of carrier synchronization for QAM receivers", Journal of VLSI Signal Processing, Kluwer academic publishers, Netherland, 2004.

[3] Asraf Mohammed Moubark, Mohd Alauddin Mohd Ali, "Simple QPSK Modulator Implemented in Virtex 6 FPGA Board for Satellite Ground Station".

[4] Shriram K Vasudevan, Sivaraman R, Z. C. Alex "Software Defined Radio Implementation", 2010.

[5] Ziyi Feng, "A SDR Implementation Using MATLAB", 2013.

[6] Praveen J, MN Shanmukha Swamy, "Minimizing Test Power In VLSI architecture Using BIST Based Low-Transition Test Pattern Generation Technique", International Journal of Electronics and Communications Engineering and Technology, Vol - 5,pp -44-52, 2014.

[7] Anton. S. Rodriguez, Michael. C. Mensinger, "Software Defined Radio Using Xilinx".

[8] Praveen J, MN Shanmukha Swamy, "BIST Based Pattern Generation For Low Power VLSI architecture", ACEEE, pp -114120, 2014.

[9] Mengduo,"Software Defined Radio: Enabling technologies and Applications", 2011.

[10] Lawrence Goeller and David Tate, "Software defined radio: Vision, Reality and current status", Cost analysis and Research division institute for defence analyses. 